\title{
Development of Carbon Rich Spin-on Sidewall Material
}

\author{
H. Yaguchi ${ }^{1}$, Dennis Shu-Hao Hsu ${ }^{2}$, D. Maruyama ${ }^{1}$, Y. Sakaida ${ }^{1}$, S. Sassa ${ }^{1}$, R. Sakamoto ${ }^{1}$, Walter \\ Wang $^{2}$, Chun-Yen Huang ${ }^{2}$, Wen-Bin Wu ${ }^{2}$, Chiang-Lin Shih ${ }^{2}$, and Bang-Ching Ho ${ }^{1}$ \\ 1: Nissan Chemical Industries, LTD. Semiconductor Materials Research Department \\ Electronic Materials Research Lab. \\ 635. Sasakura Fuchu-Machi Toyama 939-2792 Japan \\ 2: Nanya Technology Corporation, Taipei County 243-08, Taiwan, R.O.C.
}

\begin{abstract}
Sidewall process has been proposed as one of the acceptable self aligned double patterning technologies (SADPT). In this study, carbon rich spin-on sidewall (SoS) materials have been investigated. And the possibility of applying them in novel double patterning process has been explored. This process has some merits in terms of cost reduction and throughput compared with CVD sidewall process. And it is easy to apply this material for conventional litho film stacks. However, how to control critical dimension has been one of the key tasks to be dissolved. And, several issues including etch resistance and solubility must be addressed. Thus, carbon rich polymer having alkali soluble unit and cross-linking unit was chosen for this purpose. The sidewall width is controlled by the cross-link reaction using a concept similar to negative-tone photoresist. The driving force of cross-linking reaction is acid diffusion from the resist. As a result, it was confirmed that SoS pattern was formed and the critical dimension of the sidewall can be controlled by adjusting the amount of acid amplifier. By using SoS materials we successfully converted $1: 3 \mathrm{~L} / \mathrm{S}$ pattern to dense pattern.

Keyword: self aligned double patterning technologies (SADPT), spin-on sidewall material, CVD sidewall process, sidewall width, SoS pattern
\end{abstract}

\section{Introduction}

$193 \mathrm{~nm}$ immersion and hyper NA lithography are used at $45 \mathrm{~nm}$ and beyond. The next generation of lithography will use new technologies such as double pattering, EUV or EB. Particularly double patterning technology (DPT) has emerged as a leading candidate to fill the technology gap between water immersion and EUV lithography. [1-4] Various DPT approaches were proposed to achieve $3 \mathrm{X} \mathrm{nm}$ half-pitch dense lines and beyond. Three most commonly studied double patterning techniques are Litho-Etch- Litho-Etch (LELE), Litho- Processes-Litho- Etch (LPLE), and sidewall (spacer) process. From a technical viewpoint, LELE is a very promising process, but it might have the issue. The cost of ownership will be very high because LELE will cost about twice as much as the current single litho patterning process. Many device makers are exploring unique processes in order to develop a suitable double patterning technique. Flash memory makers are diligently investigating the sidewall process by CVD, which dose not need a second litho alignment step. However, the cost is still high even without a second litho step. The high cost of the CVD process will be reduced if a spin-on material is used. Therefore, we are focusing on spin-on sidewall process. The target of this process is to make $3 \mathrm{X}$ nm dense pattern.

It was reported that polymer blends and block copolymers were investigated as SoS material. In our previous study, Si-containing SoS material was chosen because its high Si-content satisfied the requirement as a hard

Received March 31, 2010

Accepted April 30, 2010 
mask for eth process. [5, 6]

In this study, we used the acid catalyzed cross-linking reaction to control sidewall width. Figure 1 shows our concept of forming SoS pattern. After coating SoS material, only the SoS material near the PR surface becomes insoluble in developer. And SoS pattern will be formed after removing the non-reaction parts of the SoS coating.

One of the goals is to reduce the cost of ownership by using spin-on coatings for the sidewall process. Particularly, the efficient approaches to control the sidewall width and profile have been investigated by the optimization of process flow and the SoS material formulation.

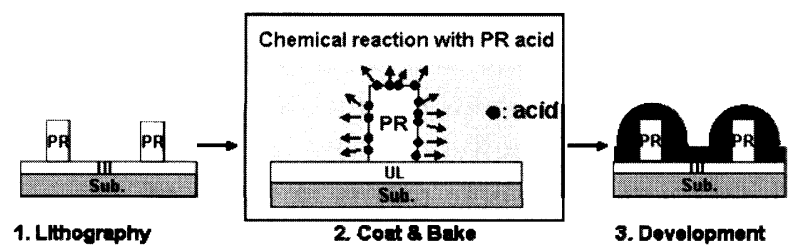

Figure 1. Scheme of proposed mechanism

\section{Experimental}

\subsection{Materials}

In this study, we considered several remarkable points including etch resistance and solubility in the top-coat solvent. SoS material used in this study contains carbon-rich polymer, cross-linker (25 wt \% relative to the polymer), acid amplifier, and 4-methyl-2-pentanol (MIBC) as a solvent. The basic components of the carbon rich polymer consist of an etch resistant core, and acidic groups for base dissolution. Molecular weight and molecular weight dispersity of the polymer were determined by gel permeation chromatographic (GPC) analysis. Polymers of different molecular weight were used in this study, as listed in Table 1. Melamin was used as a cross-linker. An acid amplifier was used as sidewall width controller. The acid amplifier is decomposed in the presence of acid catalyst, and generates sulfonic acid. The amount of acid is dramatically effected by the heating condition. The SoS material composition was listed in Table 2.
Table 1. Molecular weight and molecular weight dispersity of polymer

\begin{tabular}{ccc}
\hline C rich polymer & $\mathrm{Mw}^{a}$ & $\mathrm{Mw} / \mathrm{Mn}^{a}$ \\
\hline polymer 1 & 8,000 & 2.0 \\
polymer 2 & 15,000 & 2.0 \\
polymer 3 & 25,000 & 6.0 \\
\hline
\end{tabular}

${ }^{a}$ Molecular weight and molecular weight dispersity of polymer were determined by gel permeation chromatographic (GPC) analysis.

Table 2. Formulation of SoS material

\begin{tabular}{|c|c|c|}
\hline SoS material ${ }^{a}$ & $\begin{array}{c}\text { Crich } \\
\text { polymer }\end{array}$ & $\begin{array}{c}\text { Acid amplifier } \\
(w t \%)\end{array}$ \\
\hline 1 & polymer 1 & \\
\hline 2 & polymer 2 & \\
\hline 3 & polymer 2 & 1 \\
\hline 4 & polymer 2 & 3 \\
\hline 5 & polymer 3 & \\
\hline 6 & polymer 3 & 1 \\
\hline 7 & polymer 3 & 3 \\
\hline 8 & polymer 3 & 6 \\
\hline
\end{tabular}

${ }^{a}$ All samples contain $25 \mathrm{wt} \%$ cross-linker relative to the polymer and 4-methyl-2-pentanol (MIBC) as solvent.

\subsection{Spin-on sidewall process}

Figure 2 depicts the spin-on sidewall process flow used in this study. After forming PR patterned wafer (a), PR patterned wafers were spin-coated with SoS materials (b) in order to form SoS pattern (c). Then the top of SoS pattern and residue between lines were removed by $\mathrm{CF}_{4} / \mathrm{Ar}$ reactive ion etching, followed by $\mathrm{O}_{2} / \mathrm{N}_{2}$ etching to remove fluorine-modified surface (d). And PR pattern was dissolved by $2.38 \%$ TMAH (e). And the resulted pattern was transferred to the under layer by the subsequent etching step (f).

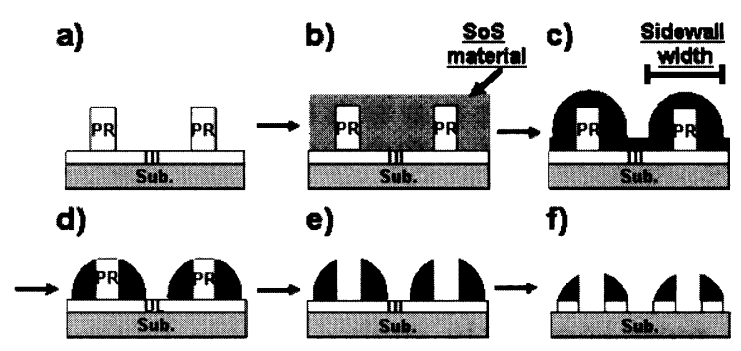

Figure 2. Carbon rich spin-on sidewall process flow used in this study 
2.3. Procedure of formation of SoS pattern and evaluation of sidewall width and profile

Three processes were used in this study (Figure 3). In all processes, $65 \mathrm{~nm}$ line PR pattern with 260 $\mathrm{nm}$ pitch $(1: 3 \mathrm{~L} / \mathrm{S})$ was used to evaluate the performance of SoS material. The initial film thickness of PR was fixed at $130 \mathrm{~nm}$. SoS film thickness was fixed at $120 \mathrm{~nm}$. And $2.38 \% \mathrm{TMAH}$ was used as developer. Sidewall width was defined as line width of sidewall pattern (Figure 2). And sidewall width was checked by CD-SEM. And sidewall profile was evaluated by cross-section SEM. Details of process condition were described in the following sentence.

\subsubsection{Baking-only process (Process (a))}

Figure 3 (a) shows a process flow of process (a). After SoS material was spin-coated on the PR patterned wafer, the wafer was baked at $150{ }^{\circ} \mathrm{C}$ for $2 \mathrm{~min}$. During the heating step, acids were generated from PR. Then non-reactive parts were removed by the developer.

\subsubsection{Process with UV exposure after coating SoS} material (Process (b))

Process (b) is described in Figure 3 (b). An exposure step was involved in order to generate acid at lower temperature. After SoS material was coated, this sample was irradiated with $248 \mathrm{~nm}$ irradiation $(\mathrm{KrF})$ and baked at $130{ }^{\circ} \mathrm{C}$ for $2 \mathrm{~min}$. Then its non-reactive parts were dissolved by the developer.

2.3.3. Process with UV exposure before coating SoS material (Process (c))

Process flow of process (c) is presented in Figure 3 (c). In this process, PR patterned wafer was irradiated before coating SoS material. $222 \mathrm{~nm}$ irradiation was used. And irradiation energy was fixed at $198 \mathrm{~mJ} / \mathrm{cm}^{2}$. Then SoS material was coated on the PR patterned wafer. And the wafer was baked at $130{ }^{\circ} \mathrm{C}$ for $2 \mathrm{~min}$. Then non-reactive parts were dissolved by the developer.

\subsection{Evaluation of solubility change}

Testing samples contain polymer 1 , cross-linker, and acid. The ratio of cross-linker was ranged from $25 \%$ to $8 \%$. The acid content was 1.56 wt $\%$ relative to the polymer. After coating these samples on the silicon wafer, the wafer was baked at deferent temperature for $1 \mathrm{~min}$, followed by developing. Undissolved film thickness was measured.

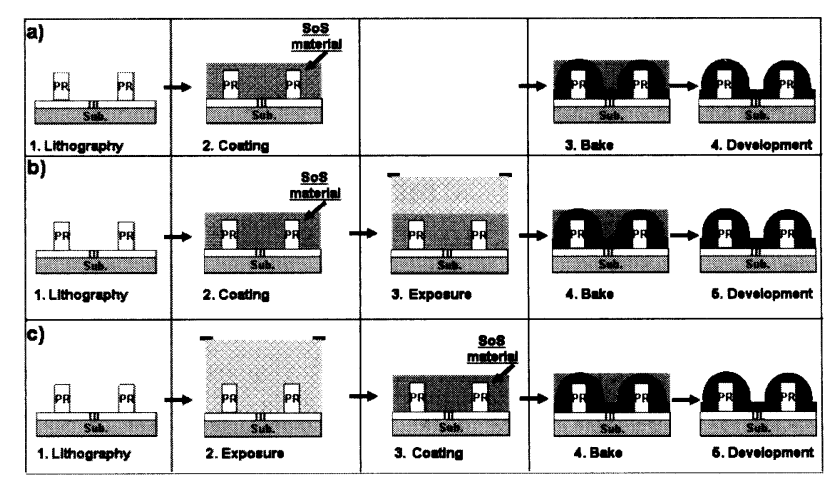

Figure 3. Process for forming SoS pattern (a: baking-only process, b: process with UV exposure after coating SoS material, c: process with UV exposure before coating SoS material)

\section{Results and Discussion}

3.1. Solubility change in developer

Figure 4 indicates the change of solubility of SoS material in the presence of acid, as heating temperature was increased from 90 to 150 deg.C. At this range of baking condition, PR profile was kept unchanged. Although samples without acid did not show any solubility change even at $150{ }^{\circ} \mathrm{C}$, all acid-containing samples became completely insoluble at $130{ }^{\circ} \mathrm{C}$. Insolubility was increased with the amounts of cross-linker.

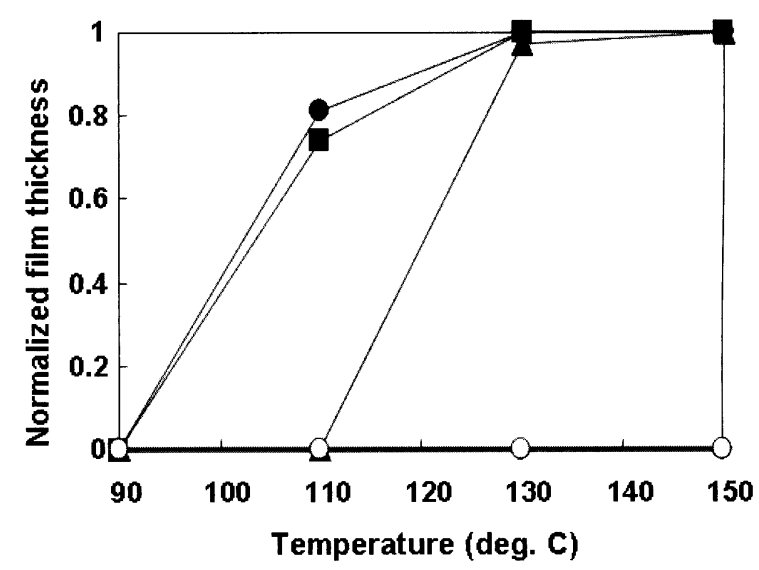

Figure 4. Solubility vs baking temperature and cross-linker content (O: 25 wt \%, 15 wt \%, $\boldsymbol{\Delta}: 8 \mathrm{wt} \%, \bigcirc: 25 \mathrm{wt} \%$ ) in terms of undissolved film thickness. These samples contain $1.56 \mathrm{wt} \%$ of acid, relative to the polymer, except for the sample of open circle $(\bigcirc)$. The initial film thickness of SoS film was fixed at $1000 \AA$.

Based on these results, the amount of cross-linker was decided to be fixed at 25 wt $\%$ relative to the polymer for latter experiments. 
3.2. Study of spin-on sidewall process and optimization of SoS material formulation

Several novel processes were studied, as shown in Figure 3. First, process (a) was evaluated as the simplest process in all processes. Second, to increase the efficiency of cross-linking reaction, acid amplifier was used, and its effect was studied. Third, an exposure step was added and its influence on acid diffusion and the sidewall width were evaluated.

\subsubsection{Baking-only process (Process (a))}

At first, process (a) was used, as shown in Figure 3(a). After PR patterned wafer was made, SoS material was coated on this substrate, and followed by baking and developing using $2.38 \%$ TMAH. Heating condition was fixed at $150{ }^{\circ} \mathrm{C}$ for $2 \mathrm{~min}$. This step is for generating acid from PR and for inducing acid catalyzed cross-linking reaction of SoS material. It was confirmed that SoS material attached to the PR surface, and the sidewall width was increased with the increase of polymer molecular weight of the SoS material (Table 3 ). In order to get wider the line width, a novel addition - acid amplifier - was introduced into SoS material. As results, the sidewall width was increased by the addition of acid amplifier (Table 3 ). But residues were generated on the space area. And the sidewall profile was degraded as the acid amplifier content increased from $1 \mathrm{wt} \%$ (3) to $3 \mathrm{wt} \%$ (4). This is because acid amplifier- containing SoS material induces

Table 3. Sidewall width obtained by using baking-only process (process (a))

\begin{tabular}{cc}
\hline SoS material & Sidewall width $(\mathrm{nm})^{a}$ \\
\hline $\mathbf{1}$ & 70 \\
$\mathbf{2}$ & 75 \\
$\mathbf{5}$ & 90 \\
$\mathbf{3}$ & 90 \\
$\mathbf{4}$ & $b$ \\
\hline
\end{tabular}

${ }^{a}$ Critical dimension of the original PR pattern: $65 \mathrm{~nm},{ }^{b}$ Not detected

thermal cross-linking reaction at $150{ }^{\circ} \mathrm{C}$ even if acid dose not come from PR, and generates the residue on the open area. If this process will be chosen. The use of thermal stable SoS material or special PR that generates acid at lower temperature are needed.

\subsubsection{Process with UV exposure after coating} SoS material (Process (b))

The purpose of adding UV exposure is to increase acid diffusion from PR for getting wider sidewall width. Table 4 shows the sidewall evaluation results with process (b). It shows increasing exposure dose could get wider sidewall width. However, higher exposure doses not only widen its sidewall width, but also worsen its profile. At 200 $\mathrm{mJ} / \mathrm{cm}^{2}$, the sidewall had good profile but the maximum obtainable sidewall width was only $90 \mathrm{~nm}$. When higher dose was used, the sidewall width was increased but its profile was no longer acceptable. Moreover, $200 \mathrm{~mJ} / \mathrm{cm}^{2}$ dose will yield very low throughput.

To get wider sidewall width with low dose is the goal for this study. The sidewall width is related to the amount of photo-generated acid and acid diffusion length. The acid amplifier was introduced in SoS material 2, as listed in Table 4. To increase the efficiency of cross-link chemical reaction between resins and cross-linkers, acid amplifier was used. These results show acid amplifier can reduce the UV exposure dose effectively. However, the sidewall width is not large enough at 40 $\mathrm{mJ} / \mathrm{cm}^{2}$. When $60 \mathrm{~mJ} / \mathrm{cm}^{2}$ was used, its profile was already deformed. And some residues were present in open area. Therefore its operational window of dose becomes too small to be practical.

Table 4. Sidewall width obtained by using process with UV exposure after coating SoS material (process (b))

\begin{tabular}{ccc}
\hline SoS material & dose $\left(\mathrm{mJ} / \mathrm{cm}^{2}\right)$ & $\begin{array}{c}\text { Sidewall width } \\
(\mathrm{nm})^{a}\end{array}$ \\
\hline $\mathbf{2}$ & 200 & 90 \\
$\mathbf{2}$ & 300 & 100 \\
$\mathbf{4}$ & 40 & 160 \\
$\mathbf{4}$ & 60 & $b$ \\
\hline
\end{tabular}

${ }^{a}$ Critical dimension of the original PR pattern: 65 nm, ${ }^{b}$ Not detected

This is because SoS material induced the photo-cross linking reaction and became insoluble in the developer.

3.2.3. Process with UV exposure before coating SoS material (Process (c))

In order to prevent SoS material from inducing the photo-cross linking reaction, the 
process was changed from process (b) to process (c). From these results, it shows the sidewall width was related to the molecular weight of the polymer (Table 5). In sample 5, acid amplifier was added. Figure 5 shows the cross-section images of the SoS patterns obtained at different acid amplifier contents for process (c). It was confirmed that sidewall width was increased with the amount of acid amplifier, and profiles were also better than other processes. For sample 8, the line width of SoS pattern was increased from $65 \mathrm{~nm}$ of the original PR CD to $180 \mathrm{~nm}$. These results show that acid amplifier is effective for getting larger sidewall width. In our study, good profiles were obtained by using process (c). This process has a lot of sidewall width controlling factors such as dose, and SoS formulation. It should be noted that it is possible to use conventional PR.

Table 5. Sidewall width obtained by using process with UV exposure before coating SoS material (process (c))

\begin{tabular}{cc}
\hline SoS material & Sidewall width $(\mathrm{nm})^{a}$ \\
\hline $\mathbf{1}$ & 101 \\
$\mathbf{2}$ & 114 \\
$\mathbf{5}$ & 120 \\
$\mathbf{6}$ & 145 \\
$\mathbf{7}$ & 150 \\
$\mathbf{8}$ & 180 \\
\hline
\end{tabular}

${ }^{a}$ Critical dimension of the original PR pattern: 65 nm

\subsection{Etching performance}

SoS pattern was prepared by using process (c), and the etching performance was evaluated according to the above procedure (Figure 2). SoS material 8 was used in this etching test. After SoS material was applied, the top of SoS pattern and the residue between $\mathrm{PR}$ lines were removed by $\mathrm{CF}_{4} / \mathrm{Ar}$ reactive ion etching, followed by $\mathrm{O}_{2} / \mathrm{N}_{2}$ etching to remove fluorine-modified surface. Then PR was removed by $2.38 \%$ TMAH. Although optimization of etching condition was needed, it was confirmed that separated patterns were obtained (Figure 6 (b)). Separated pattern width formed by SoS material and space size were $58 \mathrm{~nm}$ and 38 $\mathrm{nm}$ respectively. In order to improve the line width and the space size, etch process needs to be optimized. Meanwhile, the acid diffusion length should also be well

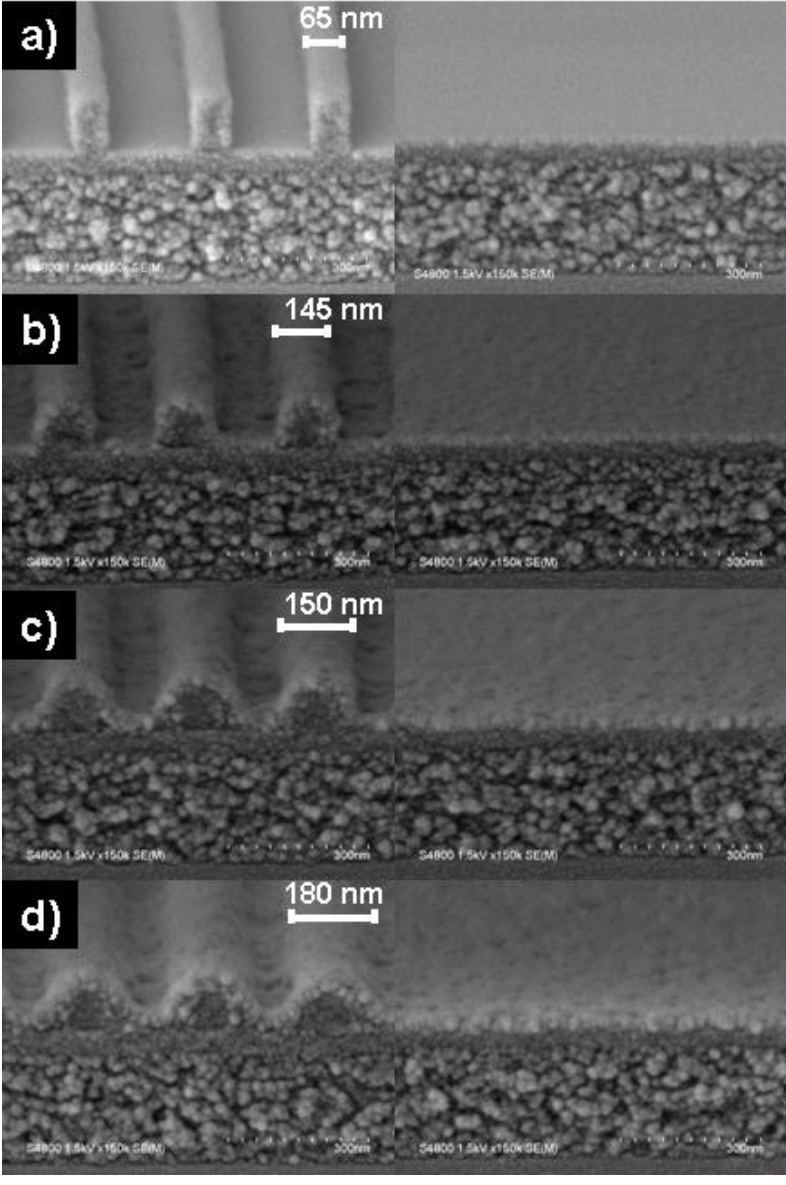

Figure 5. SoS patterns obtained from process (c) (a: original PR patterned wafer (left) and open area (right), b: after applying sample 6, c: after applying sample 7, d: after applying sample 8)

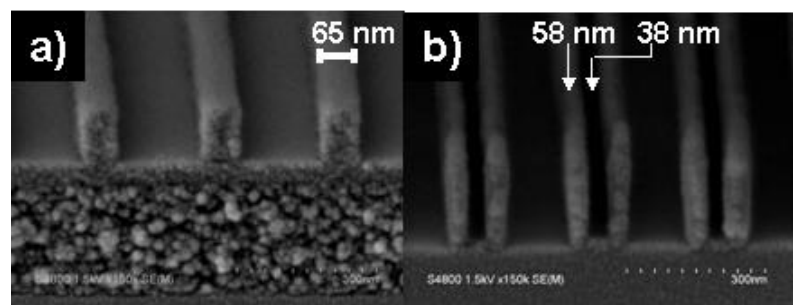

Figure 6. Cross-section SEM of original patterned wafer (a) and separated pattern formed by SoS material (b)

controlled by optimizing SoS material to reduce its line edge roughness(LER).

\section{Conclusion}

Novel SoS material was developed. This material induces a cross-linking reaction by the acid coming from PR composition, and thus attaches to the PR surface. Its sidewall width can be controlled by tuning the amounts of acid amplifier. By using the process with UV exposure before coating SoS 
material, a more suitable SoS pattern was obtained. Although the optimization of etching condition and SoS material formulation are needed, separated patterns were obtained using our spin-on sidewall process. In the future, our proposed process and SoS material are expected to form $3 \mathrm{X}-\mathrm{nm}$ half-pitch dense pattern.

\section{References}

1. M. Khojasteh, et al., "Building an immersion topcoat from the ground up: materials

perspective" Proc. SPIE, 6519, (2007), 651907.

2. M. Hori, et al., "Sub-40nm Half-Pitch Double Patterning with Resist Freezing Process" Proc. SPIE, 6923, (2008), 69230H.

3. E. Nishimura, et al., "Precise CD control techniques for double patterning and sidewall transfer" Proc. SPIE, 6924, (2008), 692425.

4. Yayi Wei, and Robert L. Brainard, "Advanced Processes for 193nm Immersion Lithography" SPIE, (2009).

5. D. Maruyama, H. Yaguchi, and Bang-Ching Ho, et al., "A cost effective Spin on Sidewall Material Alternative to the CVD Sidewall Process" SPIE Asia, (2008), 7140-116.

6. Shu-Hao Hsu, H. Yaguchi, D. Maruyama, Y. Sakaida, R. Sakamoto, Wen-Bin Wu, Chun-Yen Huang, Walter Wang, Thomas Huang, Bang-Ching Ho, and Chiang-Lin Shih, "Carbon-rich spin on sidewall material for self-aligned double- patterning technology" Proc. SPIE, 7639, (2010), 763907. 